ARTICLE

DOI: $10.1038 /$ s41467-017-01655-5

\title{
Interfaces between hexagonal and cubic oxides and their structure alternatives
}

Hua Zhou ${ }^{1,2}$, Lijun Wu ${ }^{3}$, Hui-Qiong Wang (1) 1,4, Jin-Cheng Zheng (10 1,4, Lihua Zhang ${ }^{5}$, Kim Kisslinger ${ }^{5}$, Yaping Li (1) 1, Zhiqiang Wang ${ }^{1}$, Hao Cheng${ }^{1}$, Shanming $\mathrm{Ke}^{2}, \mathrm{Yu} \mathrm{Li}^{2}$, Junyong Kang ${ }^{1}$ \& Yimei Zhu ${ }^{3}$

Multi-layer structure of functional materials often involves the integration of different crystalline phases. The film growth orientation thus frequently exhibits a transformation, owing to multiple possibilities caused by incompatible in-plane structural symmetry. Nevertheless, the detailed mechanism of the transformation has not yet been fully explored. Here we thoroughly probe the heteroepitaxially grown hexagonal zinc oxide ( $\mathrm{ZnO}$ ) films on cubic (001)magnesium oxide ( $\mathrm{MgO}$ ) substrates using advanced scanning transition electron microscopy, $X$-ray diffraction and first principles calculations, revealing two distinct interface models of (001) $\mathrm{ZnO} /(001) \mathrm{MgO}$ and (100) $\mathrm{ZnO} /(001) \mathrm{MgO}$. We have found that the structure alternatives are controlled thermodynamically by the nucleation, while kinetically by the enhanced $\mathrm{Zn}$ adsorption and $\mathrm{O}$ diffusion upon the phase transformation. This work not only provides a guideline for the interface fabrication with distinct crystalline phases but also shows how polar and non-polar hexagonal $\mathrm{ZnO}$ films might be manipulated on the same cubic substrate.

\footnotetext{
${ }^{1}$ Fujian Provincial Key Laboratory of Semiconductors and Applications, Collaborative Innovation Center for Optoelectronic Semiconductors and Efficient Devices, Department of Physics, Xiamen University, Xiamen 361005, China. ${ }^{2}$ College of Materials Science and Engineering and Shenzhen Key Laboratory of Special Functional Materials, Shenzhen University, Shenzhen 518060, China. ${ }^{3}$ Condensed Matter Physics and Materials Science Department, Brookhaven National Laboratory, Upton, NY 11973, USA. ${ }^{4}$ Xiamen University Malaysia, Sepang 43900, Malaysia. ${ }^{5}$ Center for Functional Nanomaterials, Brookhaven National Laboratory, Upton, NY 11973, USA. Hua Zhou and Lijun Wu contributed equally to this work. Correspondence and requests for materials should be addressed to H.-Q.W. (email: hqwang@xmu.edu.cn) or to J.-C.Z. (email: jczheng@xmu.edu.cn) or to Y.Z. (email: zhu@bnl.gov)
} 
ecently, in order to fabricate multifunctional electronic devices, researchers have focused on the study of interfacial structures between two materials with distinct symmetry groups $^{1-6}$, for example, one with an $\mathrm{Fm}-3 \mathrm{~m}$ (e.g. $\mathrm{MgO}$ ) or $\mathrm{Pm} 3 \mathrm{~m}$ (e.g., $\mathrm{SrTiO}_{3}$ ) space group and the other with a $P 6_{3} \mathrm{mc}$ space group (e.g., $\mathrm{ZnO}$ ). However, some issues may arise due to the change of growth directions depending on growth conditions. For example, our group ${ }^{7-9}$ and others ${ }^{10-14}$ have demonstrated that the growth direction (either polar (001) or non-polar (100)) of wurtzite $\mathrm{ZnO}$ thin films strongly depends on the substrate temperature and growth pressure. Remarkably, it has recently been observed that a change in the orientation of the coupling plane with substrates can improve device properties due to the removal of central symmetry for $\mathrm{ZnO}$. This effect is similar to the phenomenon where different planes perform different catalytic activity for the same catalyst ${ }^{15,16}$. For example, non-polar mplane $\mathrm{ZnO}$ instead of c-plane coupling with $\mathrm{ZnMgO}$ or other semiconductors can reduce the Stark effect ${ }^{17-19}$. In addition, it is beneficial to prepare high-quality films to improve the properties of the optoelectronic devices ${ }^{20,21}$. Thus, the design of coupling with different planes to improve the device properties becomes a common method for the study of $\mathrm{ZnO}$. Naturally, it is of great importance to address the issues of growth orientation and microscopic structures of the interface between different planes for the fabrications and applications of novel devices by synthesizing $\mathrm{ZnO}$ with other materials. Nevertheless, there are still lack of enough literatures to reveal the features of the microscopic structure of the interface for the different growth orientations and even less to explain the related mechanism.

In this work, firstly, we obtained a diagram of the growth orientations transformation of the $\mathrm{ZnO}$ films between the c-plane and $\mathrm{m}$-plane prepared with different growth temperatures or oxygen pressures using molecular beam epitaxy. Secondly, the microscopic structures of the interface between the $\mathrm{ZnO}$ films with wurtzite structure and the $\mathrm{MgO}$ (001) substrate with cubic structure were revealed through the scanning transmission electron microscopy (STEM) with high-angle annular dark-field (HAADF) detector and X-ray diffraction (XRD) $\phi$-scan. Finally, according to the classical nucleation theory, we propose that the physical essence of the orientation transformation (or alternative) originates from the difference of the nucleation process. The involved interface energies, surface energies, and energy change of transformation were obtained through first principles calculations based on the density functional theory (DFT) using the Vienna ab initio simulation package (VASP) code ${ }^{22,23}$. Our theoretical calculation results show that, under the condition that the contact angle of c-plane $\mathrm{ZnO}$ is much larger than $\mathrm{m}$-plane, the nucleation barrier of the $[001]_{\mathrm{ZnO}}$ orientation could be smaller than that of the $[210]_{Z n O}$ orientation. On the other hand, the higher growth temperature will induce the atom diffusion and then enhance the $\mathrm{Zn}$ atom adsorption and $\mathrm{O}$ atom diffusion on the substrate, which stimulate the transformation of growth orientation. This work not only explains the previous experimental results of the growth orientations transformation of the $\mathrm{ZnO}$ films or other materials under different growth conditions, but also paves the way to integrate optical materials of wurtzite structure (such as $\mathrm{ZnO}$, $\mathrm{GaN}$, etc) with multi-ferromagnetic and/or high dielectric materials of perovskite structure (such as $\mathrm{SrTiO}_{3}, \mathrm{BaTiO}_{3}$, etc).

\section{Results}

The diagram of growth orientation transformation. XRD results and in-situ reflection high energy electron diffraction (RHEED) patterns (Supplementary Fig. 1 and Supplementary Note 1) from the $\mathrm{ZnO}$ films demonstrate the growth orientation transformation of the film from c-plane to m-plane with the

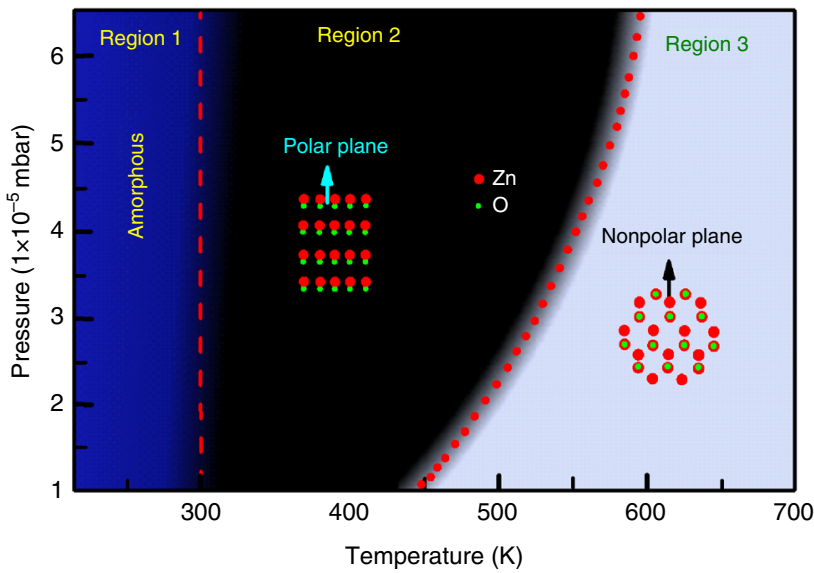

Fig. 1 Diagram of the $\mathrm{ZnO}$ growth orientations. The transformation of the growth orientation is tailored by the growth temperature and $\mathrm{O}_{2}$ partial pressure. It is similar to a conventional phase transition from water to water-vapor 22,23 or a pressure-temperature relationship diagram of the reaction $\mathrm{CaMg}\left(\mathrm{CO}_{3}\right)_{2}$ (dolomite) $+2 \mathrm{SiO}_{2}$ (coesite) $=\mathrm{CaMgSi}_{2} \mathrm{O}_{6}$ (diopside) $+2 \mathrm{CO}_{2}$ (vapor) 24

change of growth temperature and $\mathrm{O}_{2}$ pressure. A phase diagram of growth orientation as a function of temperature and $\mathrm{O}_{2}$ pressures can thus be obtained, as shown in Fig. 1. When the $\mathrm{ZnO}$ film growth occurs with the conditions in region 2 (black) or region 3 (gray), the corresponding growth orientations of the $\mathrm{ZnO}$ films grown on $(001)_{\mathrm{MgO}}$ substrates are along $[001]_{\mathrm{ZnO}}$ or $[100]^{*} \mathrm{ZnO}$ azimuth orientations, respectively (Subscripts $\mathrm{MgO}$ and $\mathrm{ZnO}$ represent $\mathrm{MgO}$ substrate and $\mathrm{ZnO}$ film, respectively. Superscript “ $*$ ” indicates the index based on reciprocal lattice). Note, $[100]^{*} \mathrm{ZnO}$ is the normal of $(100)_{\mathrm{ZnO}}$ plane, and parallel to $[210]_{\mathrm{ZnO}}$ direction. When the growth temperature is below about $300 \mathrm{~K}$ (region 1 (blue)), the $\mathrm{ZnO}$ thin film is of amorphous structure. Remarkably, this diagram of the growth direction transformation from $[001]_{\mathrm{ZnO}}$ direction to $[210]_{\mathrm{ZnO}}$ direction (as illustrated by the dotted line) is akin to a conventional phase transition from water to water-vapor ${ }^{24,25}$ or a pressuretemperature relationship diagram of the reaction $\mathrm{CaMg}\left(\mathrm{CO}_{3}\right)_{2}$ $($ dolomite $)+2 \mathrm{SiO}_{2} \quad($ coesite $)=\mathrm{CaMgSi}_{2} \mathrm{O}_{6} \quad($ diopside $)+2 \mathrm{CO}_{2}$ $(\text { vapor })^{26}$.

The relationship of the interfaces. We study the relationships between the two types of the interfaces that couple the c-and $\mathrm{m}$ planes, respectively, with the same MgO substrates using STEM, electron diffraction (ED) and XRD $\phi$-scan. Figure 2 shows the STEM images acquired by HAADF detector from the $\mathrm{c}-\mathrm{ZnO}$ film with the beam along the $\mathrm{MgO}$ [110] direction. The bright contrast dots on the left side and the weak contrast dots on the right side correspond to $\mathrm{Zn}$ and $\mathrm{Mg}$ columns, respectively, since the intensity of the STEM-HAADF image is approximately proportional to $Z^{1.7}$ ( $\mathrm{Z}$ : atomic number). There are two kinds of $\mathrm{c}-\mathrm{ZnO}$ domains. One shows a centered-rectangle like atomic arrangement, which is consistent with the $\mathrm{ZnO}$ [100] projection (Fig. 2a). The other has a close-packed atomic arrangement corresponding to a $\mathrm{ZnO}[-1-20]$ projection (Fig. 2b). From these images, we can reconstruct the interface structure between $\mathrm{ZnO}$ film and $\mathrm{MgO}$ substrate. Figure 2c shows the $\mathrm{ZnO}$ [100] (top) and [-1-20] (bottom) projection along with $\mathrm{MgO}$ [110] projection, respectively, for $\mathrm{c}-\mathrm{ZnO}$ domains I and II. Figure $2 \mathrm{~d}$ shows the top view with one $\mathrm{ZnO}$ (001) layer on one $\mathrm{MgO}$ (001) layer (marked by an orange rectangle in Fig. $2 \mathrm{c}$ ) viewed along the film normal. These 

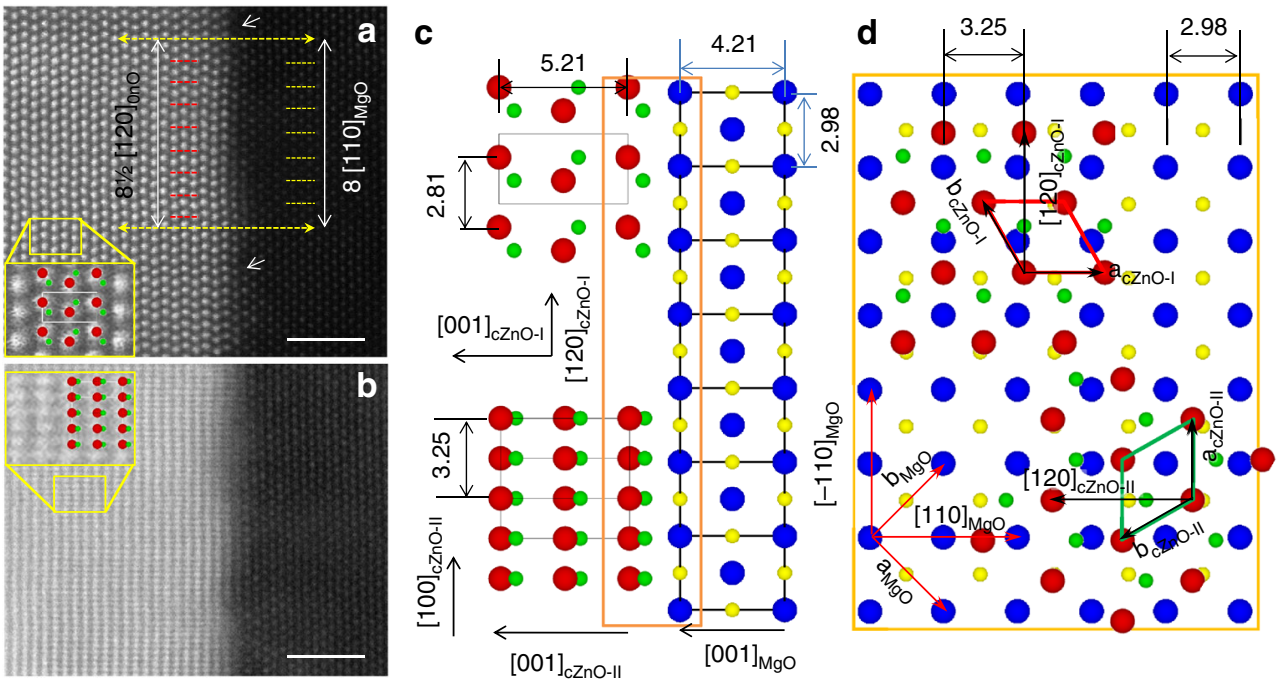

Fig. 2 Orientation relationship for c-ZnO. Cross-section scanning transmission electron microscopy (STEM)-high-angle annual dark-field (HAADF) images of (a) c-ZnO-I and (b) c-ZnO-II domains of polar film. Scale bar, $2 \mathrm{~nm}$. The beam is parallel to the [110 $]_{\mathrm{MgO}}$ direction. The high contrast dots on the left correspond to $\mathrm{Zn}$ atoms, while weak contrast dots in the right correspond to $\mathrm{Mg}$ atoms. The $\mathrm{O}$ atom is invisible due to its low atomic number. The insets show the magnified images from the area outlined by the yellow rectangles with the atomic projection of $[100]_{c Z n O-I}$ in $(\mathbf{a})$ and $[-1-20]_{c Z n O-I I}$ in $(\mathbf{b})$

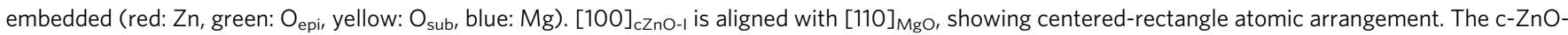
II is rotated $90^{\circ}$ along [001] direction in terms of $\mathrm{ZnO}-\mathrm{I}$ and viewed along [-1-20 $]_{\mathrm{cnnO}-\mathrm{Il}}$, showing close-packed rectangular atomic arrangement. The two yellow dashed arrows in a indicate the alignment of a $\mathrm{ZnO}$ row with respect to an $\mathrm{MgO}$ row. c Projection of the interface for c- $\mathrm{ZnO}-\mathrm{I}$ along [100] direction (upper panel) and c-ZnO-II along [-1-20] direction (lower panel) derived from STEM images. $\mathbf{d}$ Top view (one layer of (001) Zno plane on top of one layer of (001) $\mathrm{MgO}$ plane) of the interface, which is the interface area outlined by the brown rectangle in $\mathbf{c}$ (rotated $90^{\circ}$ clockwise). The unit cell of the c- $\mathrm{ZnO}-\mathrm{II}$ domain (outlined by green parallelogram) is rotated $90^{\circ}$ from that of the $\mathrm{c}-\mathrm{ZnO}-\mathrm{I}$ domain outlined by red parallelogram

STEM results reveal the interface relationships as follows:

$$
\begin{aligned}
& {[001]_{\mathrm{cZnO}-\mathrm{I}}\left\|[001]_{\mathrm{MgO}},[120]_{\mathrm{cZnO}-\mathrm{I}}\right\|} \\
& {[-110]_{\mathrm{MgO}},[100]_{\mathrm{CZnO}-\mathrm{I}} \|[110]_{\mathrm{MgO}} ;}
\end{aligned}
$$

and

$$
\begin{aligned}
& {[001]_{\mathrm{CZnO}-\mathrm{II}}\left\|[001]_{\mathrm{MgO}},[100]_{\mathrm{CZnO}-\mathrm{II}}\right\|} \\
& {[-110]_{\mathrm{MgO}},[-1-20]_{\mathrm{CZnO}-\mathrm{II}} \|[110]_{\mathrm{MgO}},}
\end{aligned}
$$

where subscripts $\mathrm{cZnO}-\mathrm{I}$ and $\mathrm{cZnO}-\mathrm{II}$ represent $\mathrm{c}-\mathrm{ZnO}$ domain I and domain II, respectively. A three-dimensional view and the related description are shown in Supplementary Fig. 2 and Supplementary Note 2, respectively. To verify the above relationships, we performed XRD $\phi$-scan experiments, as shown in Fig. 3a. There are twelve $\{011\}$ peaks over the $0-360^{\circ}$ scan, indicating two c- $\mathrm{ZnO}$ domains. The alignment of $011_{\mathrm{cZnO}-\mathrm{I}}$ peak with $-111_{\mathrm{MgO}}$ peak indicates the out-of-plane component $[001]_{\text {CZnO-II }}$ $[001]_{\mathrm{MgO}}$, and the in-plane component $[010]^{*}{ }_{\mathrm{cZnO}-\mathrm{I}} \mathrm{I}[-110]_{\mathrm{MgO}}$. Because of $[010]^{*}{ }_{\text {cZnO-I }} \|[120]_{\text {CZnO-I }}$, we obtain $[120]_{\text {CZnO-I }}$ II $[-110]_{\mathrm{MgO}}$, which agrees with the result obtained from STEM imaging. Similarly, we confirm the interface relationship for the c$\mathrm{ZnO}$ domain II. The above orientations are further confirmed by electron diffraction pattern (EDP), as shown in Fig. 3b, where the [100] zone of $\mathrm{ZnO}$ domain I and the [-1-20] zone of $\mathrm{ZnO}$ domain II are aligned with the [110] zone of the $\mathrm{MgO}$ substrate.

For $\mathrm{m}-\mathrm{ZnO}$ film ( $\mathrm{ZnO}$ (100) grown on $\mathrm{MgO}$ (001) plane), there are four pairs of $\mathrm{ZnO}(010)$ peaks over the $0-360^{\circ}$ scan, indicating that there are four $\mathrm{m}-\mathrm{ZnO}$ domains (Fig. 3c). One pair of 010 peaks, which are $180^{\circ}$ apart (labeled in black text $010_{\text {mZnO-I }}$ and $1-10_{\mathrm{mZnO}}$-I with subscript $\mathrm{mZnO}-\mathrm{I}$ representing $\mathrm{m}$ - $\mathrm{ZnO}$ domain I), deviate $301^{\circ}$ (or $360^{\circ}-301^{\circ}=59^{\circ}$ ) and $121^{\circ}$, respectively, from the $111_{\mathrm{MgO}}$ peak. This indicates that their in-plane components $[-120]^{*}{ }_{\mathrm{mZnO}-\mathrm{I}}$ (or $[010]_{\mathrm{mZnO}-\mathrm{I}}$ in real-lattice) and
$[1-20]^{*}{ }_{\mathrm{mZnO}-\mathrm{I}}$ (or $[0-10]_{\mathrm{mZnO}-\mathrm{I}}$ ) deviate $59^{\circ}$ and $121^{\circ}$, respectively, from the in-plane component $[110]_{\mathrm{MgO}}$ of $\mathrm{MgO}$. Because the angle between $\mathrm{MgO}$ [4-10] and [110] is 59 $[010]_{\mathrm{mZnO}-\mathrm{I}}$ is parallel to $[4-10]_{\mathrm{MgO}}$. Moreover, as the angle between $\mathrm{ZnO}[011]$ and $[010]$ is about $58^{\circ},[011]_{\mathrm{mZnO}-\mathrm{I}}$ is roughly parallel to $[110]_{\mathrm{MgO}}$ (just $1^{\circ}$ difference). Therefore, when the sample is tilted to the $[110]_{\mathrm{MgO}}$ zone, the $[011]_{\mathrm{mZnO}-\mathrm{I}}$ zone should be observed, and is shown in Fig. 3d using EDP taken from this domain. The above orientation relationship is straightforward in our STEM-HAADF observations, as shown in Fig. 4. The first one shows a center-rectangle pattern with dumbbell-like dots, corresponding to the $\mathrm{ZnO}$ [011] projection (Fig. 4a). The STEM-HAADF image calculated based on the multislice method (inset with white outline in Fig. 4a) agrees with the observation very well, confirming the orientation of $\mathrm{ZnO}$ [011]. Although the $\mathrm{Mg}$ atoms in the $\mathrm{MgO}$ substrate (right in Fig. 4a) are well resolved, their shape is slightly elongated vertically, indicating that the $\mathrm{MgO}$ substrate slightly deviates from the [110] zone. A simulation with $\mathrm{MgO}$ deviating from the [110] zone by $1^{\circ}$ (embedded in Fig. 4a, outlined by green lines) agrees with the experimental image very well. When $\mathrm{ZnO}$ [011] deviates from $\mathrm{MgO}[110]$ by $1^{\circ}$, its [010] is parallel to [4-10] $]_{\mathrm{MgO}}$, which is consistent with the XRD $\phi$-scan result. On the basis of this, we can reconstruct the interface structure between the $\mathrm{m}-\mathrm{ZnO}$ film and the $\mathrm{MgO}$ substrate, as shown in the top of Fig. $4 \mathrm{c}(\mathrm{ZnO}$ [011] projection along with $\mathrm{MgO}$ [110] projection) and Fig. $4 \mathrm{~d}$ (viewed along the film normal with one layer of $\mathrm{ZnO}$ (100) on one layer of $\left.(001)_{\mathrm{MgO}}\right)$. A 3D view is also shown in Supplementary Fig. 2. The orientation relationship is as follows:

$$
\begin{aligned}
& (100)_{\mathrm{mZnO}-\mathrm{I}}\left\|(001)_{\mathrm{MgO}},[010]_{\mathrm{mZnO}-\mathrm{I}}\right\| \\
& {[4-10]_{\mathrm{MgO}},[001]_{\mathrm{mZnO}-\mathrm{I}} \|[140]_{\mathrm{MgO}} .}
\end{aligned}
$$

Another pair of (010) peaks in Fig. 3c (labeled as black text 

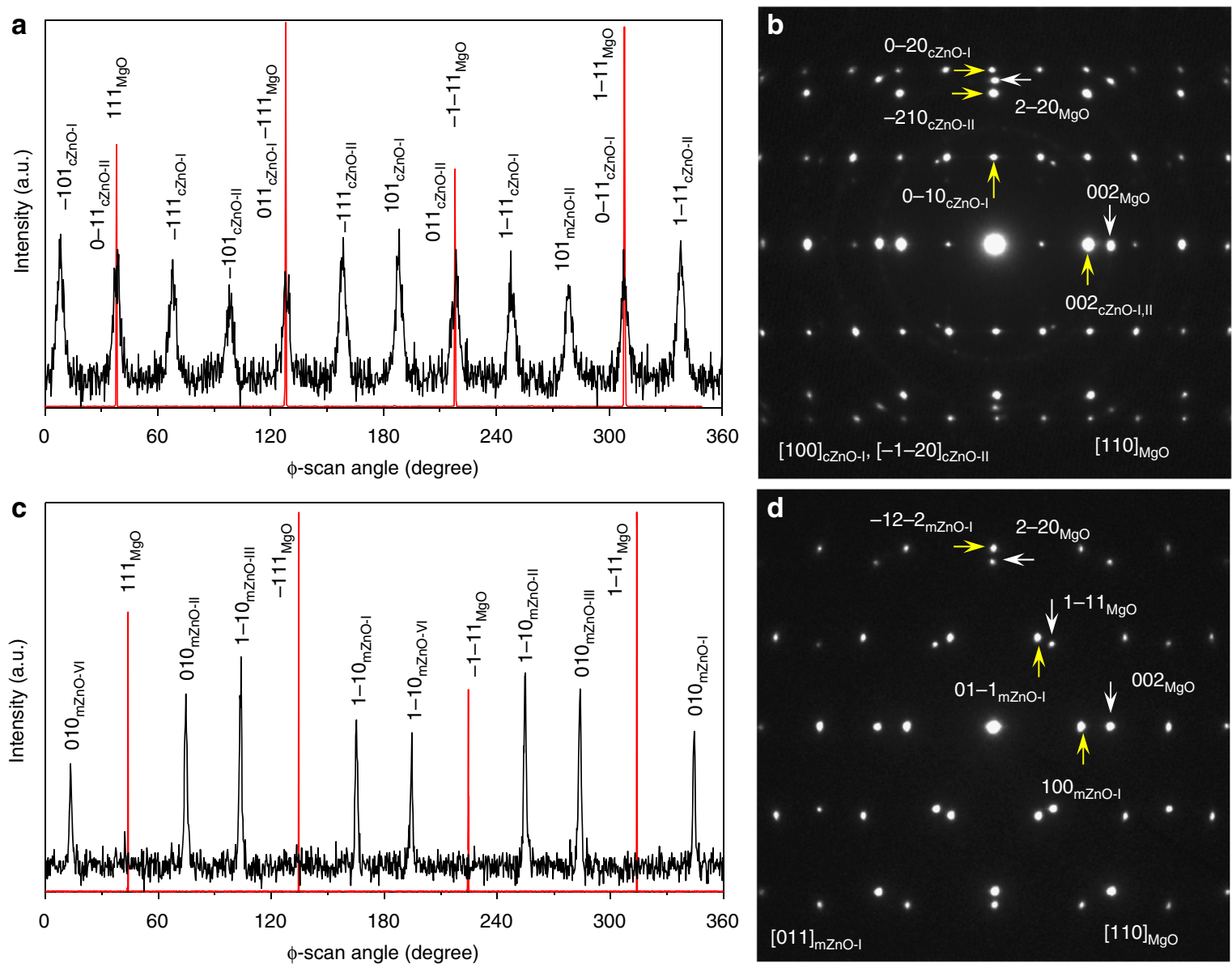

Fig. 3 Orientation relationship for $\mathrm{c}-\mathrm{ZnO}$ and $\mathrm{m}-\mathrm{ZnO}$. a X-ray diffraction (XRD) $\phi$ scan and (b) Electron diffraction pattern (EDP) from the c-ZnO film. The $\mathrm{XRD} \phi$-scan is carried out with $2 \theta=37^{\circ}, \chi=54.7^{\circ}$ for $\mathrm{MgO}$ and $2 \theta=36.2^{\circ}, \chi=61.6^{\circ}$ for $\mathrm{c}-\mathrm{ZnO}$. The EDP consists of three sets of patterns: $[110]_{\mathrm{MgO}}$, $[100]_{\mathrm{cZnO}-I}$ and $[-1-20]_{\mathrm{c}-\mathrm{ZnO}-\mathrm{II}}$ (subscripts $\mathrm{MgO}, \mathrm{cZnO}-\mathrm{I}$, and cZnO-II represent $\mathrm{MgO}$ substrate, c-ZnO domain I and II, respectively). c XRD $\phi$-scan and (d) [011] zone EDP from the $\mathrm{m}-\mathrm{ZnO}$ film. Subscripts $\mathrm{MgO}, \mathrm{mZnO}-\mathrm{I}, \mathrm{mZnO}-\mathrm{II}, \mathrm{mZnO}-\mathrm{III}$, and $\mathrm{mZnO}-\mathrm{VI}$ represent $\mathrm{MgO}, \mathrm{m}-\mathrm{ZnO}$ domains I, II, III, IV, respectively. The XRD $\phi$-scan is carried out with $2 \theta=37^{\circ}, \chi=54.7^{\circ}$ for $\mathrm{MgO}$ and $2 \theta=31.7^{\circ}, \chi=60^{\circ}$ for $\mathrm{m}$ - $\mathrm{ZnO}$

$010_{\mathrm{mZnO}-\mathrm{II}}$ and $1-10_{\mathrm{mZnO}-\mathrm{II}}$ with subscript $\mathrm{mZnO}-\mathrm{II}$ representing $\mathrm{m}-\mathrm{ZnO}$ domain II) deviate from the $111_{\mathrm{MgO}}$ peak by $31^{\circ}$ and $211^{\circ}$, respectively, indicating that $[010]_{\mathrm{mZnO}-\mathrm{II}}$ is parallel to $[140]_{\mathrm{MgO}}$. Actually, the $\mathrm{m}-\mathrm{ZnO}-\mathrm{II}$ is rotated $90^{\circ}$ along $[210]_{\mathrm{ZnO}}$ direction (the normal of (001) plane) in terms of $\mathrm{m}-\mathrm{ZnO}-\mathrm{I}$. In this case, the $\mathrm{ZnO}$ [08-3] is parallel to $\mathrm{MgO}$ [110]. Therefore, when the incident beam is along the $\mathrm{MgO}$ [110] direction, the $\mathrm{m}-\mathrm{ZnO}$ II would be viewed along a high index zone $\left([08-3]_{\mathrm{mZnO}-\mathrm{II}}\right)$ in which the projected distances among the atoms along $[011]_{\mathrm{mZnO}}$ II (vertical direction) are too close to be resolved. In this way, the streak-like pattern with a spacing of about $0.262 \mathrm{~nm}$ is formed. The calculated STEM-HAADF image along [08-3] direction (inset with white outline in Fig. 4b) agrees with the observation very well. The reconstructed interface structure based on XRD and STEM-HAADF is shown in the bottom part of Fig. $4 \mathrm{c}, \mathrm{d}$. The second orientation relationship is:

$$
\begin{aligned}
& (100)_{\mathrm{mZnO}-\mathrm{II}}\left\|(001)_{\mathrm{MgO}},[010]_{\mathrm{mZnO}-\mathrm{II}}\right\| \\
& {[140]_{\mathrm{MgO}},[001]_{\mathrm{mZnO}-\mathrm{II}} \|[-410]_{\mathrm{Mgo}} .}
\end{aligned}
$$

From the third and the fourth pairs of 010 peaks in Fig. 3c, we conclude the third and the fourth orientation relationships for the
$\mathrm{m}-\mathrm{ZnO}$ film:

$$
\begin{aligned}
& (100)_{\mathrm{mZnO}-\mathrm{III}}\left\|(001)_{\mathrm{MgO}},[010]_{\mathrm{mZnO}-\mathrm{III}}\right\| \\
& {[1-40]_{\mathrm{MgO}},[001]_{\mathrm{mZnO}-\mathrm{III}} \|[410]_{\mathrm{MgO}} \cdot} \\
& (100)_{\mathrm{mZnO}-\mathrm{VI}}\left\|(001)_{\mathrm{MgO}},[010]_{\mathrm{mZnO}-\mathrm{VI}}\right\| \\
& {[410]_{\mathrm{MgO}},[001]_{\mathrm{mZnO}-\mathrm{VI}} \|[-140]_{\mathrm{MgO}} \cdot}
\end{aligned}
$$

Here, subscripts $\mathrm{mZnO}$-III and $\mathrm{mZnO}-\mathrm{VI}$ represent $\mathrm{m}-\mathrm{ZnO}$ domains III and VI, respectively. The calculated STEM-HAADF images along with reconstructed interfacial structures are shown in Supplementary Fig. 3 with discussions in Supplementary Note 3. When the film is viewed along the MgO [110] direction for STEM-HAADF imaging, the $\mathrm{m}$ - $\mathrm{ZnO}$ domain III looks identical to the $\mathrm{m}-\mathrm{ZnO}$ domain $\mathrm{I}$, while the $\mathrm{m}-\mathrm{ZnO}$ domain $\mathrm{VI}$ looks identical to $\mathrm{m}-\mathrm{ZnO}$ domain II. These results can be further confirmed by the in-situ RHEED patterns (Supplementary Fig. 4 and Supplementary Note 4). Therefore, although XRD 0-360 $\phi$ scan reveals four $\mathrm{m}-\mathrm{ZnO}$ domains, STEM-HAADF imaging would only distinguish two of them.

The observation of four $\mathrm{m}-\mathrm{ZnO}$ domains is consistent with $4 \mathrm{~mm}$ symmetry along [001] of the substrate $\mathrm{MgO}$. Eight $\mathrm{MgO}$ $<410>$ are equivalent, while $\mathrm{ZnO}[010]$ and $[0-10]$ are 

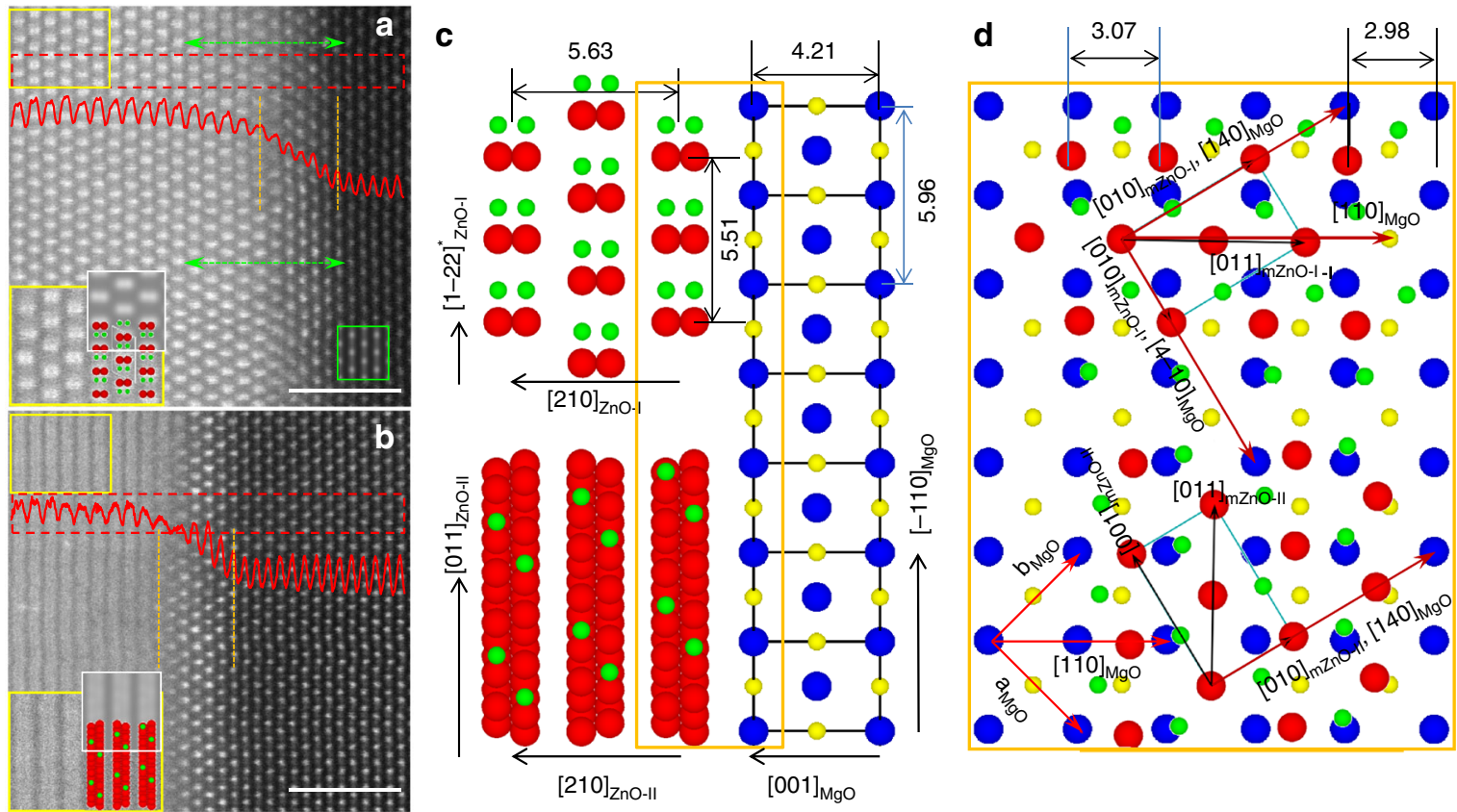

Fig. 4 Orientation relationship for $\mathrm{m}$-ZnO. Scanning transmission electron microscopy (STEM)- high-angle annual dark-field (HAADF) images of (a) m$\mathrm{ZnO}-\mathrm{I}$ and $(\mathbf{b}) \mathrm{m}-\mathrm{ZnO}-\mathrm{II}$ domains of the m-plane film. Scale bar, $2 \mathrm{~nm}$. The insets show the magnified images from the area outlined by the yellow rectangles with the atomic projection of [011 $]_{\mathrm{mZnO}-1}$ in $\mathbf{a}$ and $[0-83]_{\mathrm{mZnO}-I}$ in $\mathbf{b}$ embedded. The inset outlined by white line squares in $\mathbf{a}, \mathbf{b}$ are calculated STEM-HAADF images along $[011]_{\mathrm{ZnO}}$ and $[08-3]_{\mathrm{ZnO}}$, respectively. They agree with the experimental images very well. Note, there are $5 \sim 6$ atomic layers in the interface that have an atomic arrangement similar to $\mathrm{MgO}$, as marked by a pair of orange vertical dashed lines. The image intensity decreases from the $\mathrm{ZnO}$ film to the $\mathrm{MgO}$ substrate over these layers, as indicated by the embedded image intensity line scans (red) from the red dashed rectangles (integrated vertically). This indicates that there exists an interface phase $\mathrm{Zn}_{x} \mathrm{Mg}_{1-x} \mathrm{O}$ ( $x$ decreases from the $\mathrm{ZnO}$ film side to the $\mathrm{MgO}$ substrate side, see Supplementary Note 9 for electron energy loss spectroscopy (EELS) analysis). The two green dashed arrows in a indicate the alignment of a $\mathrm{ZnO}$ row with an $\mathrm{MgO}$ row. c Projection of the interface for $\mathrm{m}-\mathrm{ZnO}-\mathrm{I}$ along [011] direction (upper panel) and $\mathrm{m}$-ZnO-II along [08-3] direction (lower panel) derived from STEM images. d Top view (one layer of a $(100)_{\mathrm{ZnO}}$ plane on top of one layer of a (001) $\mathrm{MgO}$ plane) of the interface area outlined by the brown rectangle in $\mathbf{c}$ (rotated $90^{\circ}$ clockwise). The $\mathrm{m}-\mathrm{ZnO}-\mathrm{II}$ domain (lower panel) can be obtained by rotating $90^{\circ}$ from $\mathrm{m}-\mathrm{ZnO}-\mathrm{I}$ (upper panel)

equivalent. There would be equal probability for $\mathrm{ZnO}$ to align its [010] (or [0-10]) parallel to one of the $\mathrm{MgO}<410>$, resulting in the formation of four independent $\mathrm{m}-\mathrm{ZnO}$ domains. The occurrence of two rotational domains in $\mathrm{c}-\mathrm{ZnO} / \mathrm{MgO}$ and four rotational domains in $\mathrm{m}-\mathrm{ZnO} / \mathrm{MgO}$, which is consistent with the theoretical prediction based on the mismatch of rotational symmetry at the interface (Supplementary Note 5). The observed interface relationship for $\mathrm{m}-\mathrm{ZnO}$ shown in (Eqs. 3-6), as well as that of $\mathrm{c}-\mathrm{ZnO}$ shown in (Eqs. 1 and 2), is confirmed by the first principles calculations to have the lowest interface energy among a range of the possible interface structures (see later discussions).

Lattice coupling between the film and the substrate. The $d$-spacing of $\mathrm{MgO}$ along $<110>$ direction is equal to $2.98 \AA$, while those of $\mathrm{ZnO}$ along [010] ${ }^{*}$ (or [120]) and [2-10]* (or [100]) are equal to $2.81 \AA$ and $1.62 \AA$, respectively. The lattice mismatch is, therefore, calculated to be $5.7 \%$ along the $[120]_{\mathrm{ZnO}}$ direction and $8.7 \%$ along the $[100]_{\mathrm{ZnO}}$ direction in the case of $\mathrm{c}-\mathrm{ZnO}$. From Fig. 2a, the lattice coupling relationship between the film and the substrate at the interface for c- $\mathrm{ZnO}$ is: $17 \mathrm{~d}_{010 \mathrm{ZnO}}=16 \mathrm{~d}_{110 \mathrm{MgO}}$ (or $81 / 2[120]_{\mathrm{ZnO}}=8[110]_{\mathrm{MgO}}$, see horizontal dashed lines in Fig. $2 \mathrm{a}$ ). In addition, from the STEM-HAADF image (Fig. 2a) and EDP (Fig. 3b), the lattice parameters $a$ and $c$ were measured to be 3.24 $\pm 0.02 \AA$ and $5.22 \pm 0.02 \AA$, respectively, in the films. These are close to the values of bulk sample ( $a=3.25 \AA$ and $c=5.21 \AA)$. That is to say, the lattice parameter $a_{\mathrm{ZnO}}$ appears almost unchanged from the bulk to the film. For the c-ZnO film, the termination at the interface could be either oxygen-plane or zincplane. To determine the termination, we simultaneously acquired
STEM-HAADF and annular bright field (ABF) images for c- $\mathrm{ZnO}$, as shown in Supplementary Fig. 5. The insets show the magnified images from the area outlined by the rectangles with the atomic projection of $[100]_{\text {cZnO-I }}$. While $\mathrm{O}$ contrast is weak and blur near the interface due to the strain, they are clearly seen in the right side of the $\mathrm{Zn}$ atoms in the magnified image, indicating that the $\mathrm{ZnO}$ film is along [001] direction, e.g., $\mathrm{O}$ is closer to the interface than $\mathrm{Zn}$, which is consistent with our first principle calculations that the O-termination at the interface yields a lower interface energy (see discussions later). Supplementary Fig. 5 shows inverse FFT images by applying an aperture in $020_{\mathrm{ZnO}} /-220_{\mathrm{MgO}}$ spots of the FFT (inset) of STEM-HAADF image. Dislocations are clearly seen at the interface, as marked by T. From the simultaneously acquired STEM-HAADF and STEM-ABF images for c-ZnO film (Supplementary Fig. 5) with the interface on the right, sharper O contrast in the ABF image can be seen as the film in this area is free of defects. More detailed discussions can be found in Supplementary Note 6 and Supplementary Fig. 6, and Supplementary Note 7 shows that c-plane films are composed of two different kinds of 'nanorods' with a rotation of $30^{\circ}$ between them (the corresponding boundary lines are marked by the red lines). These observations suggest the $\mathrm{c}-\mathrm{ZnO}$ films are formed by the selfassembly growth model. The defects located at the interface between the $\mathrm{ZnO}$ layer and $\mathrm{MgO}$ layer as marked by the white arrows in Fig. 2a can be further confirmed by the examination of the enhanced contrast with color and the plot of peak intensities in 3D and profile, as shown in Supplementary Fig. 7 and descripted in Supplementary Note 8 . Clemens et al. ${ }^{29}$ demonstrated that there were many point defects on the MgO-(001) plane after the treatment of annealing owing to the $\mathrm{Mg}$ atoms 
evaporation. But, it is beneficial for the nucleation with the 3-dimensional island model.

For the $\mathrm{m}-\mathrm{ZnO}$ film, the lattice mismatch between the film and the substrate is about $7.4 \%$ and $5.5 \%$ along the $[-110]_{\mathrm{MgO}}$ and $[110]_{\mathrm{MgO}}$ directions, respectively. The lattice coupling relationship between the film and the substrate at the interface is: $13 \mathrm{~d}_{1-22 \mathrm{ZnO}}=6 \mathrm{~d}_{110 \mathrm{MgO}}$ (see the atom rows bounded by the two green dashed arrows in Fig. 4a). From this and EDP in Fig. 3d, the $\mathrm{d}$-spacing of $\mathrm{ZnO}$ along [1-22]* direction (in-plane) and [100]* (out-of-plane) were measured to be $1.375 \pm 0.02 \AA$ and $2.816 \pm$ $0.02 \AA$, respectively, close to the corresponding bulk values $(1.378$ and $2.815 \AA$ ) too. The result that the lattice lengths are nearly equal to the bulk values can be further confirmed by the in-situ RHEED patterns (Supplementary Fig. 8 and Supplementary Note 9). Furthermore, from the STEM-HAADF image, the interface of $\mathrm{c}-\mathrm{ZnO}$ is quite sharp, indicating an absence of diffuse areas of the substrate to $\mathrm{ZnO}$ film in the $\mathrm{c}-\mathrm{ZnO}$ case. While in the case of $\mathrm{m}-\mathrm{ZnO}$, there is a transition area which has an atomic arrangement similar to $\mathrm{MgO}$, but with stronger contrast, as marked by the orange dashed lines in Fig. 4 and Supplementary Fig. 6. This indicates there exists a transition phase (denoted as $\mathrm{T}$-phase) of $\mathrm{Zn}_{x} \mathrm{Mg}_{1-x} \mathrm{O}$. This result had been confirmed by the EELS spectra (Supplementary Figs. 9 and 10, and Supplementary Notes 10 and 11). Apparently, the T-phase has a structure similar to cubic $\mathrm{MgO}$, rather than $\mathrm{ZnO}$. First principles calculations based on DFT results imply that the existence of T-phase on the $\mathrm{MgO}$ substrate will induce the decrements of the substrate surface energy, $\mathrm{O}$ diffusion barrier as well as the barrier to nucleation while increasing the $\mathrm{Zn}$ atom adsorption energy (see later discussions). To sum up, from the discussion about the STEMHAADF images, the values of lattice parameter in films are close to the bulk value in spite of the existence of the large lattice mismatch and the interface for $\mathrm{c}-\mathrm{ZnO}$ is sharper than that for $\mathrm{m}-\mathrm{ZnO}$.

First principles calculations. In this section, in order to investigate the mechanism of the transformation of the growth orientation and the stability of the interface structure between the $\mathrm{ZnO}$ film and the MgO (001) substrate, we carried out the first principle calculations based on DFT for the interface structures of two growth orientations. The corresponding calculation details can be found in the Supplementary Notes 12-16.

For polar plane of $\mathrm{ZnO}$, it is "cleavage energy" instead of "surface energy" that can be defined as the cleavage of the bulk $\mathrm{ZnO}$ creates both (001)-Zn surface and (00-1)-O surface and no unique surface energy can be determined ${ }^{28,29}$. Here the "surface energy" is taken as half of the cleavage energy (using the values in Supplementary Table 2). The calculated "surface energy" values for c-, m- and a-plane of $\mathrm{ZnO}$ as well as the (001) $\mathrm{MgO}$ substrate surface are in good agreement with previously reported results also calculated by the DFT + GGA method ${ }^{29-32}$. For interface energy calculations, two interface models for c- $\mathrm{ZnO}$ with either O-termination or Zn-termination at the interface are considered and the calculations show a lower interface energy with $\mathrm{O}$ termination than that with Zn-termination, in agreement with our experimental observation that the O-layer is closer to the $\mathrm{MgO}$ substrate (Supplementary Fig. 5). For the $\mathrm{m}-\mathrm{ZnO}$ case, two interface models are also considered with and without the buffer layers between hexagonal $\mathrm{ZnO}$ and cubic $\mathrm{MgO}$; for the model with buffer layer, the change of the substrate surface energy is considered as induced by the buffer layers, using a range between that of the (001) MgO and that of the (001)FCC ZnO, leading to a range of interface energy mentioned in Supplementary Note 14.

In order to understand why the experimentally observed interface registry follows the relationship as shown in (Eqs. 1 and
2) for $\mathrm{c}-\mathrm{ZnO} / \mathrm{MgO}$ and (Eqs. 3-6) for $\mathrm{m}-\mathrm{ZnO} / \mathrm{MgO}$, we compared the total energies of a range of possible interface registry structures for the two growth orientations through theoretical calculations by the DFT method. Setting the experimentally observed registry structure as the origin point (orientation degree equals to $0^{\circ}$ ), a series of other possible registry structures can be obtained by the clockwise (anti-clockwise) rotation of the $\mathrm{ZnO}$ overlayers along the surface normal of the $\mathrm{MgO}$ substrate, defined for negative (positive) rotation degrees as shown in Supplementary Fig. 11. The blue and red curves plot the calculated related energies (using the $0^{\circ}$ orientation as the zero energy reference) for $\mathrm{c}-\mathrm{ZnO}$ and $\mathrm{m}-\mathrm{ZnO}$, respectively, for the possible interface registry structures from $-15^{\circ}$ to $15^{\circ}$ rotations and demonstrate that the interfacial relationships observed in our experiments can yield the lowest interface energies. This confirms why we have obtained the robust interfacial relationships for $\mathrm{ZnO} / \mathrm{MgO}$ interfaces.

Supplementary Fig. 12 show the adsorption energies for the $\mathrm{Zn}$ and $\mathrm{O}$ atoms (corresponding to the most stable saddle-point configurations shown in Supplementary Fig. 13), respectively, on the $\mathrm{MgO}$ (001) surface without and with the buffer layer. Remarkably, the adsorption energy for $\mathrm{Zn}$ atoms (higher than $-0.1 \mathrm{eV}$ ) is far higher than that for $\mathrm{O}$ atoms (lower than $-4.0 \mathrm{eV}$ ) on the $\mathrm{MgO}(001)$ surface without the buffer layer, indicating that it is rather easy for $\mathrm{Zn}$ atoms to desorb, and hence difficult to form an island with $\mathrm{Zn}$ atom as the termination layer of the $\mathrm{ZnO}$ film close to the interface. On the contrary, $\mathrm{O}$ atoms can adsorb solidly on the MgO substrate. When there exist a buffer layer on the $\mathrm{MgO}$ surface, the $\mathrm{Zn}$ atom adsorption energy becomes higher, as shown in Supplementary Fig. 12, indicating that the formation of the transition layer can hinder the $\mathrm{Zn}$ desorption. Whereas, the buffer layer has little impact on $\mathrm{O}$ adsorption energies. The diffusion barriers for $\mathrm{Zn}$ and $\mathrm{O}$ atoms (as shown in Supplementary Fig. 12) show that $\mathrm{Zn}$ atom diffusion barrier is rather small (lower than $0.025 \mathrm{eV}$ ) no matter whether there exists a transition phase or not on the $\mathrm{MgO}$ substrate. However, for $\mathrm{O}$ atoms, the diffusion barrier on the ideal $\mathrm{MgO}$ surface is much larger (about1.2 eV) and becomes smaller (about0.8 eV) after adding the buffer layer(s).

\section{Discussions}

As experimentally demonstrated, the grown $\mathrm{ZnO}$ films on the $\mathrm{MgO}$ (001) substrates can exhibit either polar $c$ - or non-polar $m$ directions depending on the growth conditions. The growth direction of the film was robust and exhibited a transition after nucleating, which can be demonstrated by in-situ annealing experiments (Supplementary Fig. 14 and Supplementary Note 17) under the higher temperature condition (around $673 \mathrm{~K}$ ) with or without $\mathrm{O}_{2}$. In fact, nucleation of the $\mathrm{ZnO}$ film on the $\mathrm{MgO}$ substrate from the gas phase is intrinsically a non-equilibrium phenomenon governed by a competition between kinetics and thermodynamics. Next, we discuss the mechanisms of the growth orientation alternatives from the viewpoints of thermodynamics and kinetics based on the first principles calculation results.

On the basis of the classical theory of nucleation ${ }^{33-35}$, for nuclei in shape of clusters or islands that can be described in terms of macroscopic surface energy ${ }^{36}$, the overall excess free energy $\Delta G$ for nucleation islands of $\mathrm{ZnO}$ on the $\mathrm{MgO}$ substrate can be obtained using the following equations

$$
\begin{aligned}
\Delta G= & S_{\text {interface }} \cdot\left(\gamma_{\text {interface }}-\gamma_{\text {substrate }}\right) \\
& +S_{\text {island }} \cdot \gamma_{\text {island }}+V_{\text {island }} \cdot \Delta G_{v}
\end{aligned}
$$

where $S_{\text {interface }}$ is the interface area of contact between the nucleation island and the substrate, $S_{\text {island }}$ and $V_{\text {island }}$ are the surface area and volume of nucleation island, respectively. 
$\gamma_{\text {interface }}$ represents the interface energy per unit area, and $\gamma_{\text {substrate }}$ and $\gamma_{\text {island }}$, are the surface energy per unit area for substrate and island, respectively. $\Delta G_{v}$ is the free energy change of the island per unit volume. The values of interface energy $\left(\gamma_{\text {interface }}\right)$, surface energy $\left(\gamma_{\text {substrate }}\right)$ and free energy change $\left(\Delta G_{v}\right)$ can be obtained from first principles calculations (Supplementary Tables 1-3), whereas, $\gamma_{\text {island }}$ depends on the shape of the island.

As shown by the atomic force microscopy (AFM) images in Supplementary Fig. 15), the island slope of the nucleation stage for $\mathrm{c}-\mathrm{ZnO}\left(8^{\circ}-14^{\circ}\right)$ is much greater than for $\mathrm{m}-\mathrm{ZnO}\left(1^{\circ}-4^{\circ}\right)$ after growing for $10 \mathrm{~min}$. Therefore, the growth of the $\mathrm{c}-\mathrm{ZnO}$ follows a three-dimensional-like mode, while that of $\mathrm{m}-\mathrm{ZnO}$ is of a 2dimensional-like mode. Additionally, the corresponding in-situ RHEED patterns, which show rather weak spots for the $\mathrm{c}-\mathrm{ZnO}$ and yet bright stripes for the $\mathrm{m}-\mathrm{ZnO}$, available in the insets of Supplementary Fig. 15, show that the roughness of $\mathrm{c}-\mathrm{ZnO}$ is far greater than that of $\mathrm{m}-\mathrm{ZnO}$, further confirming the difference of the contact angle of nucleation between $\mathrm{c}-\mathrm{ZnO}$ and $\mathrm{m}-\mathrm{ZnO}$.

On the basis of AFM images, the morphology of c- $\mathrm{ZnO}$ and $\mathrm{m}-$ $\mathrm{ZnO}$ islands is estimated to be of typical polygonal shape, i.e., "frustum with facets" (with typical contact angles of $11^{\circ}$ for c$\mathrm{ZnO}$ and $3^{\circ}$ for $\mathrm{m}-\mathrm{ZnO}$ ). However, it is not straightforward to obtain reliable shapes of small islands by AFM, due to convolution effects with the tip shape, therefore, we have also considered two additional possible shapes for the islands, namely "circular cone frustum" and "spherical cap" (Supplementary Figs. 16-18 and discussions in Supplementary Notes 18-21). Remarkably, all the three proposed morphological shapes lead to the same conclusion that, $\mathrm{c}-\mathrm{ZnO}$ with a larger contact angle yields a smaller nucleation barrier than that of $\mathrm{m}-\mathrm{ZnO}$ with a smaller contact angle. Therefore, the nucleation barrier seems to be more sensitive to the contact angle than the exact shape of the nuclei. The theoretical analysis is in good agreement with our experimental observation that $\mathrm{c}-\mathrm{ZnO}$ films with a larger contact angle are grown at a lower temperature while $\mathrm{m}-\mathrm{ZnO}$ films with a smaller contact angle exhibit at a higher temperature.

From the calculation results (Supplementary Figs. 11 and 12), we can find that during nucleating, the limit factor is adatom adsorption for $\mathrm{Zn}$ atoms and yet adatom diffusion for $\mathrm{O}$ atoms. Here, the buffer layers (the so-called T-phase) play a vital role for the adsorption energy and diffusion barrier. At a lower growth temperature, there appear no T-phase on the substrate and most of $\mathrm{O}$ adatoms adsorb solidly on the $\mathrm{MgO}$ surface with only little diffusion. Simultaneously, little $\mathrm{Zn}$ atoms can adsorb on the ideal $\mathrm{MgO}$ surface. Under this condition, there will appear nucleation with O-termination and with growth direction along [001] as well as a large contact angle. This interface feature with a rigid termination is similar to the self-assembled organic molecular nailing down the gold nanostructures ${ }^{37}$. On the contrary, if the growth temperature becomes higher, it will induce the atom diffusions and then form the $\mathrm{T}$-phase on the $\mathrm{MgO}$ substrate, which hinders the desorption of $\mathrm{Zn}$ atoms and promotes the diffusion of $\mathrm{O}$ atoms. This result will stimulate the simultaneous presentation of $\mathrm{Zn}$ and $\mathrm{O}$ atoms on the termination layer of the $\mathrm{ZnO}$ film at the interface and thus decrease the contact angle between the nucleation and $\mathrm{MgO}$ substrate. Thereby the increment of growth temperature as well as the appearance of the Tphase will result in the occurrence of the growth orientation transformation. Additionally, the increment of $\mathrm{O}_{2}$ partial pressure can inhibit the atom diffusion, and simultaneously rise the probability of the nucleation with O-termination. This can explain why even for the higher growth temperature (up to $600 \mathrm{~K}$ ), the growth orientation of $\mathrm{ZnO}$ film still appears along the [001] azimuth with the increase of $\mathrm{O}_{2}$ partial pressure (Fig. 1).

In summary, this work examines the mechanism of the growth orientation transformation. The transformation is tailored by the growth temperature and pressure simultaneously, resembling the phase transition of water to vapor-water. The interface structures of both $\mathrm{c}-\mathrm{ZnO} / \mathrm{MgO}$ and $\mathrm{m}-\mathrm{ZnO} / \mathrm{MgO}$ were thoroughly characterized by XRD, EDP, and STEM as well as first principles calculations based on DFT, revealing two rotational domains for the $\mathrm{c}-\mathrm{ZnO}$ films and four rotational domains for the $\mathrm{m}-\mathrm{ZnO}$ films and their interface registry stability. The mechanism of the growth orientation is examined from both thermodynamics and kinetics points of view. Thermodynamically, it is found that, the nucleation barrier for $\mathrm{c}-\mathrm{ZnO}$ with a larger contact angle (e.g., $\geq 11^{\circ}$ ) is lower than that of $\mathrm{m}-\mathrm{ZnO}$ with a smaller contact angle (e.g., $\leq 4^{\circ}$ ). Kinetically, it is found that, under the condition of lower growth temperature or larger $\mathrm{O}_{2}$ pressure, with few $\mathrm{Zn}$ atoms adsorbing and few $\mathrm{O}$ atoms diffusing on the substrate, the nucleation pathway follows a three-dimensional model with a large contact angle and an O-termination at the interface, resulting in the growth along the $c$-plane direction. On the contrary, at a higher growth temperature, the $\mathrm{Zn}$ atom adsorption and $\mathrm{O}$ atom diffusion are both enhanced, which promotes the transformation of nucleation model from a 3-dimensional to a qausi-two-dimensional model, resulting in the growth along the m-plane direction. Both thermodynamic and kinetic mechanisms explain the diagram of growth direction transformation tailored by the growth temperature and pressure, as shown in Fig. 1. This work not only offers a clear image of the interfacial coupling between the cubic and wurtzite phase, but also proposes a reasonable theory model to explain the phenomena of the growth orientation transformations.

\section{Methods}

The $\mathrm{ZnO}$ thin films were prepared on $\mathrm{MgO}$ (001) substrates by molecular beam epitaxy (MBE). Before being transferred into the MBE chamber for $\mathrm{ZnO}$ thin films growth, the $\mathrm{MgO}$ substrates were cleaned in an ultrasonic bath sequentially with acetone and ethanol for $5 \mathrm{~min}$, respectively. Prior to the film growth, the $\mathrm{MgO}$ substrates were thermally cleaned at about $750 \mathrm{~K}$ for $60 \mathrm{~min}$, with an oxygen pressure of $5 \times 10^{-5} \mathrm{mbar}$ and a plasma power of $250 \mathrm{w}$. As shown in our previous report ${ }^{7}$, the bright and streaky in-situ RHEED patterns from the treated MgO (001) surface indicated a smooth surface structure. In order to investigate the differences in the morphology between the two films with different growth directions, two series of ZnO films (namely polar and non-polar planes, respectively) were prepared. The polar films were grown at a substrate temperature of about $450 \mathrm{~K}$ and $\mathrm{Zn}$ (with a purity of $99.9999 \%$ ) source temperature of $630 \mathrm{~K}$ with $1 \times 10^{-5} \mathrm{mbar}$ of the oxygen pressure and $180 \mathrm{~W}$ of the plasma power. For the non-polar $\mathrm{ZnO}$ films the corresponding growth conditions are about $650 \mathrm{~K}$ (substrate temperature), $630 \mathrm{~K}$ ( $\mathrm{Zn}$ source temperature), $1 \times 10-{ }^{5} \mathrm{mbar}$ (oxygen pressure) and $180 \mathrm{~W}$ (plasma power), respectively. The surface structures and the interface relationships between the films and substrates were analyzed by in-situ RHEED and ex-situ XRD techniques (using a Rigaku rotating anode X-ray generator and diffractometer). The interfacial atomic structure for the $\mathrm{c}-\mathrm{ZnO}$ and $\mathrm{m}-\mathrm{ZnO}$ films were examined by STEM-HAADF images, electron diffraction patterns and EELS spectra using double aberration corrected JEM-200CF microscope equipped with Gatan quantum energy filter and dual EELS. Image simulations were carried out using our own computer codes based on the multislice method with frozen phonon approximation. Finally, the growth mechanism of the $\mathrm{ZnO}$ thin films was studied through analyzing the evolution of the surface morphology by AFM.

Calculation methods. In this paper, we performed first principle calculations based on $\mathrm{DFT}^{22}$ through VASP to examine the interface stability, including the calculations of surface energy, interface energy for two different growth orientations with a variety of interface structure for each orientation and compare with the observations from the STEM, RHEED and XRD results. The exchange and correlation effects were treated by the generalized gradient approximation $(\mathrm{GGA})^{23}$. Projected augmented wave (PAW) potentials were employed with the cutoff of $500 \mathrm{eV}$ for all the calculations. The EELS spectra were calculated using the TELNES package included in the WIEN2K $\operatorname{code}^{38}$, a full potential linear augmented planewave plus local-orbitals method within DFT.

Data availability. The data that support the findings of this study are available from the corresponding authors upon request. 
Received: 27 August 2016 Accepted: 6 October 2017

Published online: 14 November 2017

\section{References}

1. Schulze, F. et al. Crystallographic and electric properties of MOVPE-grown AlGaN/GaN-based FETs on Si (l0 0 1) substrates. J. Cry. Growth 299, 399-403 (2007).

2. Dadgar, A. et al. Epitaxy of GaN on silicon-impact of symmetry and surface reconstruction. New. J. Phys. 9, 389 (2007).

3. Brandt, M. et al. Ferroelectric thin film field-effect transistors based on $\mathrm{ZnO} /$ $\mathrm{BaTiO}_{3}$ heterostructure. J. Vac. Sci. Technol. B 27, 1789-1793 (2009).

4. Liu, Z. W., Sun, C. W., Gu, J. F. \& Zhang, Q. Y. Epitaxial relationship of ZnO film with $\mathrm{Si}(001)$ substrate and its effect on growth and morphology. Appl. Phys. Lett. 88, 251911 (2006).

5. Bera, A. et al. A Versatile light-switchable nanorod memory: wurtzite $\mathrm{ZnO}$ on perovskite $\mathrm{SrTiO}_{3}$. Adv. Funct. Mater. 23, 4977-4984 (2013).

6. Game, O., Singh, U., Kumari, T., Banpurkar, A. \& Ogale, S. ZnO (N)-SpiroMeOTAD hybrid photodiode: an efficient self-powered fast-response UV (visible) photosensor. Nanoscale 6, 503-513 (2014).

7. Zhou, H. et al. Wurtzite $\mathrm{ZnO}$ (001) films grown on cubic $\mathrm{MgO}$ (001) with bulklike opto-electronic properties. Appl. Phys. Lett. 99, 141917 (2011).

8. Zhou, H. et al. Tailoring of polar and nonpolar $\mathrm{ZnO}$ planes on $\mathrm{MgO}(001)$ substrates through molecular beam Epitaxy. Nanoscale Res. Lett. 7, 184 (2012).

9. Zhou, H. et al. Evolution of wurtzite $\mathrm{ZnO}$ Films on cubic $\mathrm{MgO}$ (001) Substrates: a structural, optical, and electronic investigation of the misfit structures. ACS Appl. Mater. Interfaces 6, (13823-13832 (2014).

10. Fujimura, N., Nishihara, T., Goto, S., Xu, J. F. \& Ito, T. Control of preferred orientation for ZnOx films: control of self-texture. J. Crys. Growth 130, 269-279 (1992).

11. Cagin, E. et al. Growth and structural properties of m-plane $\mathrm{ZnO}$ on $\mathrm{MgO}$ (001) by molecular beam epitaxy. Appl. Phys. Lett. 92, 233505 (2008).

12. Scarisoreanu, N. et al. Properties of $\mathrm{ZnO}$ thin films prepared by radio-frequency plasma beam assisted laser ablation. Appl. Surf. Sci. 247, 518-525 (2005).

13. Seo, S. H. \& Kang, H. C. The crossover of preferred orientation in heteroepitaxial $\mathrm{ZnO} / \mathrm{MgO}$ (001) films. J. Crys. Growth 326, 166-170 (2011).

14. Deng, R. et al. Surface morphology, structural and optical properties of polar and non-polar $\mathrm{ZnO}$ thin films: A comparative study. J. Crys. Growth 311, 4398-4401 (2009).

15. Zhou, K. B., Wang, X., Sun, X. M., Peng, Q. \& Li, Y. D. Enhanced catalytic activity of ceria nanorods from well-defined reactive crystal planes. J. Catal. 229, 206-212 (2005).

16. Hu, L. H., Peng, Q. \& Li, Y. D. Selective synthesis of $\mathrm{Co}_{3} \mathrm{O}_{4}$ nanocrystal with different shape and crystal plane effect on catalytic property for methane combustion. J. Am. Chem. Soc. 130, 16136-16137 (2008).

17. Chauveau, J. M. et al. Non-polar a-plane $\mathrm{ZnMgO} / \mathrm{ZnO}$ quantum wells grown by molecular beam epitaxy. Semicond. Sci. Technol. 23, 035005 (2008).

18. Lin, W. H., Wu, J. J., Chou, M. M. C. \& Chang, L. W. Growth and characterization of nonpolar $(10-10) \mathrm{Zn}_{1-\mathrm{x}} \mathrm{Mg}_{\mathrm{x}} \mathrm{O}(0<\mathrm{x}<0.113)$ epitaxial films: a comparison of $\gamma-\mathrm{LiAlO}_{2}$ (100) and sapphire (1010) substrates. Cryst. Growth Des. 9, 3302 (2009).

19. Chen, J. J., Deng, X. R. \& Deng, H. Progress in the growth and characterization of nonpolar $\mathrm{ZnO}$ films. J. Mater. Sci. 48, 532 (2013).

20. Taïnoff, D. et al. Residual and nitrogen doping of homoepitaxial nonpolar mplane $\mathrm{ZnO}$ films grown by molecular beam epitaxy. Appl. Phys. Lett. 98, 131915 (2011).

21. Zhang, H. H. et al. The role of band alignment in p-type conductivity of Nadoped ZnMgO: Polar versus non-polar. Appl. Phys. Lett. 104, 112106 (2014).

22. Perdew, J. P. \& Zunger, A. Self-interaction correction to density-functional approximations for many-electron systems. Phys. Rev. B 23, 5048-5079 (1981).

23. Perdew, J. P., Burke, K. \& Ernzerhof, M. Generalized gradient approximation made simple. Phys. Rev. Lett. 77, 3865-3868 (1996).

24. Bridgman, P. W. The phase diagram of water to $45,000 \mathrm{~kg} / \mathrm{cm}^{2}$. J. Chem. Phys. 5, 964-966 (1937).

25. Robert, W. L. Experimental determination of the reaction dolomite +2 coesite $=$ diopside $+2 \mathrm{CO}_{2}$ to $6 \mathrm{GPa}$. Contrib. Mineral Petrol. 122, 152 (1995).

26. Bridgman, P. W. The Pressure-volume-temperature relations of the liquid, and the phase diagram of heavy water. J. Chem. Phys. 3, 597 (1935).

27. Clemens, B. \& Claude, R. H. Atomic resolution imaging of the (001) surface of UHV cleaved $\mathrm{MgO}$ by dynamic scanning force microscopy. Phys. Rev. Lett. 91, $196102(2003)$
28. Wander, A. et al. Stability of polar oxide surface. Phys. Rev. Lett. 86, 3811 (2001).

29. Meyer, B. \& Marx, D. Density-functional study of the structure and stability of ZnO surfaces. Phys. Rev. B 67, 035403 (2003).

30. Claeyssens, F. et al. Growth of $\mathrm{ZnO}$ thin films-experiment and theory. J. Mater Chem. 15, 139 (2005).

31. Skorodumova, N. V., Hermansson, K. \& Johansson, B. Structural and electronic properties of the (100) surface and bulk of alkaline-earth metal. Phys. Rev. B 72, 125414 (2005).

32. Broqvist, P., Grönbeck, H. \& Panas, I. Surface properties of alkaline earth metal oxides. Surf. Sci. 554, 262-271 (2004).

33. Mullin, J. W. Crystallization. 4th edition, (Butterworth-Heinemann, Oxford, 2001).

34. Kalikmanov, V. I. Nucleation Theory (Springer, Dordrecht, 2013).

35. Oxtoby, D. W. Nucleation of first-order phase transitions. Acc. Chem. Res. 31 , 91-97 (1998)

36. Venables, J. A., Spiller, G. D. T. \& Hanbucken, M. Nucleation and growth of thin films. Rep. Prog. Phys. 47, 399-459 (1984).

37. Jadzinsky, P. D., Calero, G., Ackerson, C. J., Bushnell, D. A. \& Kornberg, R. D. Structure of a Thiol monolayer-protected gold nanoparticle at $1.1 \AA$ Resolution. Science 318, 430-433 (2007).

38. Blaha, P. et al. WIEN2K, An Augmented Plane Wave + Local Orbitals Program For Calculating Crystal Properties (Technische Universitat Wein, Wein, 2001).

\section{Acknowledgements}

This work is supported by the National Natural Science Foundation of China (Grant Nos. U1332105, 61227009, and 91321102), the Fundamental Research Funds for Central Universities (Grant No. 20720160020). The electron microscopy work at the Condensed Matter Physics and Materials Science Department, BNL was supported by the U.S. Department of Energy, Office of Basic Energy Science (BES), Materials Science and Engineering Division, and work at the Center for Functional Nanomaterials was supported by BES Scientific User Facilities Division, both were under Contract No. DE-SC0012704.

\section{Author contributions}

H.Z. Carried out the growth and characterization experiments, performed the first principle calculations and drafted the manuscript. H-Q.W.: Led the project, analyzed the results, and revised the manuscript. L.W. and Y.Z.: Led the STEM/EELS experiments, data analysis and orientation relationship determination. J-C.Z.: Led the theoretical calculations and nucleation model analysis. L.W. and Y.L.: Analyzed the XRD data. Z.W., H.C., S.K., and Y.L.: Participated in the modeling and simulations. L.Z. and K.K.: Prepared the TEM samples and captured high resolution TEM images. J.K.: Provided guidelines and discussions for the MBE experiments.

\section{Additional information}

Supplementary Information accompanies this paper at doi:10.1038/s41467-017-01655-5.

Competing interests: The authors declare no competing financial interests.

Reprints and permission information is available online at http://npg.nature.com/ reprintsandpermissions/

Publisher's note: Springer Nature remains neutral with regard to jurisdictional claims in published maps and institutional affiliations.

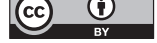

Open Access This article is licensed under a Creative Commons Attribution 4.0 International License, which permits use, sharing, adaptation, distribution and reproduction in any medium or format, as long as you give appropriate credit to the original author(s) and the source, provide a link to the Creative Commons license, and indicate if changes were made. The images or other third party material in this article are included in the article's Creative Commons license, unless indicated otherwise in a credit line to the material. If material is not included in the article's Creative Commons license and your intended use is not permitted by statutory regulation or exceeds the permitted use, you will need to obtain permission directly from the copyright holder. To view a copy of this license, visit http://creativecommons.org/ licenses/by/4.0/.

(C) The Author(s) 2017 